\title{
PRESUNTA PARÁLISIS PERIÓDICA HIPERCALÉMICA CON MIOTONÍA EN PERROS SHI-TZÚ Y PASTOR ALEMÁN
}

Suspected hyperkalemic periodic paralysis with myotonia in Shi Tzu and German Shepherds dogs

\section{Pellegrino, F.C.}

Facultad de ciencias veterinarias. Universidad de Buenos Aires. Chorroarín 280 (1427) - Ciudad Autónoma de Buenos Aires, Argentina.

Autor para correspondencia: Fernando Pellegrino. fernando.pellegrino2@gmail.com.ar

Historial del artículo:

Recibido: 1 de febrero de 2019

Aceptado: 8 de enero de 2020

\section{RESUMEN}

En los humanos, las canalopatías del músculo esquelético son entidades de base genética y de incidencia muy baja. En relación al canal de sodio en particular, las diferentes mutaciones $S C N 4 A$ pueden dar lugar a diversos fenotipos que van desde la parálisis muscular (parálisis periódica hipercalémica, parálisis periódica hipocalémica) a la hiperexcitabilidad muscular (paramiotonía congénita, miotonías agravadas por potasio), pasando por fenotipos combinados de las dos anteriores. Existe una estrecha interrelación entre la parálisis periódica hipercalémica y las miotonías; ambas características pueden coexistir en el mismo paciente.

Este trabajo describe un cuadro clínico que afectó a una camada de 7 cachorros Pastor Alemán y a una camada de 4 cachorros Shi-Tzú, con episodios caracterizados por un fenotipo clínico de parálisis muscular combinado con hiperexcitabilidad muscular.

De acuerdo a los hallazgos clínicos (fenomenología clínica, edad de aparición, recurrencia de los episodios, ausencia de signos clínicos entre los episodios) y de laboratorio (hipercalemia), y a los resultados del tratamiento con acetazolamida, el diagnóstico presuntivo fue parálisis periódica hipercalémica con miotonía/ paramiotonía por una canalopatía SCN4A.

Destacamos la importancia del diagnóstico clínico para estas raras patologías. En ausencia de diagnóstico genético, los métodos de diagnóstico complementario tiene valor limitado debido al carácter funcional de estos trastornos.

Proponemos esta comunicación como la primera de este tipo en perros de raza Pastor Alemán y Shi-Tzú.

Palabras clave: Perros; Neurología; Canalopatías de Músculo Esquelético; Parálisis Periódica Hipercalémica; Miotonía/Paramiotonía. 


\section{SUMMARY}

In humans, skeletal muscle channelopathies are genetic disease with very low incidence. Regarding the sodium channel in particular, the different SCN4A mutations can result in various phenotypes ranging from muscle paralysis (hyperkalemic periodic paralysis, hypokalemic periodic paralysis) to muscle hyperexcitability (congenit paramyotonia, myotonias aggravated by potassium), through combined phenotypes of the previous two. There is a close interrelation between hyperkalemic periodic paralysis and myotonias; both characteristics can coexist in the same patient.

This work describes a clinical picture affecting a litter of 7 German Shepherd puppies and a litter of 4 ShihTzu puppies with episodes characterized by a clinical phenotype of muscular paralysis combined with muscular hyperexcitability.

Based on clinical findings (clinical phenomenology, age of onset, episodic recurrence, absense of clinical signs in the period between episodes) and laboratory (hyperkalemia), and the results of treatment with acetazolamide, the presumptive diagnosis was hyperkalemic periodic paralysis with myotonia/paramyotonia due to SCN4A channelopathy.

We emphasize the importance of clinical diagnosis for these rare pathologies. In absence of genetic diagnosis, complementary diagnostic methods have limited value due to the functional nature of these disorders.

We proposed this description as the first communication of this type in German Shepherd and Shi-Tzu dogs.

Key words: Dogs; Neurology; Skeletal Muscle Channelopathies; Hyperkalemic Periodic Paralysis; Myotonia/Paramyotonia.

\section{INTRODUCCIÓN}

La Parálisis Periódica Hipercalémica (PPHiperC) es una canalopatía del músculo esquelético (CME), causada por alteraciones del canal de sodio. Aunque el signo cardinal lo constituyen los episodios de debilidad muscular con flacidez, puede adoptar 3 manifestaciones clínicas diferentes: a) sin miotonía; b) con miotonía clínica o electromiográfica; o c) con paramiotonía (Camacho 1984; Ricker et al. 1986; Wagner et al. 1997; Kim et al. 2001; Okuda et al. 2001; Renner y Ptacek 2001; Brancati et al. 2003; Jurkat-Rott y Lehmann-Horn 2010; Cannon 2015; 2018; Lowrie y Garosi 2017). En los humanos, los episodios comienzan en la primera década de vida y progresan hasta la pubertad, momento en el que el curso de la enfermedad se estabiliza. Posteriormente, a partir de los 40 años, la frecuencia y gravedad de los episodios disminuye sustancialmente (Zapata-Wainberg et al. 2015). En los pacientes que presentan mio- tonía, los signos disminuyen con el ejercicio (Rüdel y Lehmann-Horn 1985), en oposición a aquellos con paramiotonía, en los que la rigidez muscular se incrementa con la actividad repetida (Cannon 2015).

De acuerdo a la clasificación de miotonías propuesta en medicina veterinaria, la PPHiperC se encuentra incluida en el grupo de las Miotonías No Distróficas (MND). Constituyen un conjunto heterogéneo de trastornos poco frecuentes, causados por canalopatías genéticas de sodio o de cloro. Las miotonías por mutaciones del canal de cloro incluyen la Miotonía Congénita (MC) del Schnauzer miniatura y de otras razas (Pastor Ganadero Australiano y Terrier de Jack Russel). Las miotonías por mutaciones del canal de sodio incluyen la Paramiotonía Congénita (PMC), la PPHiperC y las Miotonías Agravadas por Potasio (MAP) (Lowrie y Garosi 2017). Esta clasificación es la que utilizaremos en este artículo.

El complejo canal de sodio se expresa en las membranas plasmáticas del músculo esque- 
lético, tanto en los túbulos transversos como en el sarcolema. Los canales se activan rápidamente $(<1 \mathrm{mseg})$ en respuesta a la despolarización, y producen un gran influjo de sodio, que resulta en una rápida carrera ascendente del potencial de acción. El canal de sodio que predomina en el músculo esquelético es un heterodímero de la subunidad $\mathrm{Na}_{\mathrm{v}} 1.4 \alpha$ formadora de poros (Trimmer et al. 1989) y la subunidad $\beta_{1}$ no-covalente asociada (Isom et al. 1992). La subunidad $\mathrm{Na}_{\mathrm{v}} 1.4 \alpha$ está codificada por el gen SCN4A en el cromosoma 17q23 (George et al. 1992), y la subunidad $\beta_{1}$ por el gen $S C N 1 B$ en el cromosoma 19q13.11 (McClatchey et al. 1992). Las mutaciones de la subunidad $\mathrm{Na}_{\mathrm{v}} 1.4 \alpha$ producen una variedad de fenotipos clínicos musculoesqueléticos, en los que la excitabilidad muscular puede estar patológicamente aumentada o reducida (Cannon 2015; Lehmann-Horn et al. 2004). Las mutaciones SCN4A que incrementan la excitabilidad del sarcolema alteran la cinética del canal provocando un aumento de su función. De esta manera impiden la inactivación del canal de sodio y causan un influjo persistente de la corriente de este ion (Cannon 2018; Jurkat-Rott et al. 2015; Kim 2014; Statland et al. 2018). Una despolarización moderada permite a los canales de sodio normales oscilar entre la recuperación de la inactivación y la reactivación de los canales mutantes, lo que se traduce en disparos repetitivos de potenciales de acción que pueden llevar a miotonía (Jurkat-Rott y Lehmann-Horn 2010; Kim 2014; Jurkat-Rott et al. 2015; Zapata-Wainberg et al. 2015; Cannon 2018; Statland et al. 2018). La despolarización prolongada de la membrana incrementa la actividad de los canales de potasio dependientes de voltaje, lo que amplifica el eflujo de potasio desde la célula muscular e incrementa los niveles de potasio sérico provocando hipercalemia (Tricarico y Camerino 2011). La disminución de la excitabilidad muscular también ocurre por aumento de la función del canal en el segmento que actúa como sensor de voltaje en las subunidades $\mathrm{Na}_{\mathrm{v}} 1.4$ mutantes, creando un poro de activación patógeno a través del cual los cationes se filtran en estado de reposo. Estos poros se activan muy rápidamente y no poseen un mecanismo de inactivación específico, generando una corriente interna de sodio muy pequeña (corriente de poro de activación), que hace a las miofibras susceptibles de una despolarización aberrante en respuesta a niveles de potasio reducidos (Cannon 2015; 2018; Spillane et al. 2016). La despolarización hace a la membrana refractaria a producir potenciales de acción. La manifestación clínica son episodios de parálisis muscular flácida, en asociación con concentraciones séricas bajas de potasio. La causa más común es la PPHipoC, con ataques recurrentes de moderada a severa debilidad (Lehmann-Horn et al. 2004; Cannon 2015). La miotonía nunca ocurre en estos casos y es un criterio de exclusión para el diagnóstico (Cannon 2018).

Las mutaciones del gen SCN4A fueron identificadas como causales de enfermedad mediante cartografía genética (Ptacek et al. 1991a; 1993) y análisis mutacional (Ptacek et al. 1991b, 1993; Rojas et al. 1991) en familias humanas afectadas. Se han identificado más de 70 mutaciones $\mathrm{Na}_{\mathrm{v}} 1.4$ en pacientes humanos con trastornos musculoesqueléticos (Huang et al. 2017). La mayoría de ellas son mutaciones de sentido erróneo que alteran la función del canal (Zaharieva et al. 2016). Entre las mutaciones que causan MND y CME, algunas son recurrentes y pueden ser utilizadas como herramienta molecular para el diagnóstico. Es el caso de las mutaciones del canal de sodio T704M y M1592V (Cannon 2002; Jurkat-Rott et al. 2002; Renner y Ptacek 2001), así como también R672H (Jurkat-Rott et al. 2000; Sternberg et al. 2001), que causan PPHiperC y PPHipoC, respectivamente. En adición a estas mutaciones clásicas, que están asociadas con fenotipos clínicos bien caracterizados (Rüdel et al. 1993; Miller et al. 2004), existen otras más raras y únicas para una familia en particular, que pue- 
den causar tipos inusuales de canalopatías de sodio (Vicart 2004).

De acuerdo a las características de los canales mutantes, las canalopatías de sodio se manifiestan como un espectro de desórdenes que oscilan desde la miotonía hasta la parálisis flácida, con fenotipos clínicos que comparten ambas características en el mismo paciente. De este modo, se han descrito una variedad de subtipos estrechamente relacionados, todos ellos causados por mutaciones de los canales de sodio, cuyas características se superponen con PPHipoC y PPHiperC, incluyendo la PP normocalémica (PPnormoC), la PMC y las MAP (miotonía fluctuante, miotonía permanente y miotonía sensible a la acetazalomida) (Okuda et al. 2001; Jurkat-Rott et al. 2015; Cannon 2018; Statland et al. 2018). Las manifestaciones clínicas varían desde síndromes miotónicos puros (MAP) a trastornos que cursan primariamente con miotonía y episodios de debilidad (por ej., PMC), o trastornos con debilidad episódica primaria con miotonía ocasional (PPHiperC y PPnormoC) (Cannon 2015,2018; Statland et al. 2018). Hay un gran solapamiento entre las distintas enfermedades, y determinar si se trata, por ejemplo, de PMC o PPHiperC depende fundamentalmente de los signos clínicos predominantes (Jurkat-Rott et al. 2002, 2015; Zapata-Wainberg et al. 2015; Statland et al. 2018).

Las opciones de tratamiento consisten en cambios del estilo de vida, minimizando los estímulos desencadenantes de las MND y, si es necesario, tratamiento sintomático de la miotonía. Los pacientes con PMC pueden aliviarse evitando la exposición al frío, y los pacientes con PPHiperC, PMC o MAP pueden hacerlo evitando las comidas ricas en potasio. La utilización de inhibidores de la anhidrasa carbónica (en particular acetazolamida y diclorfeniramina) es de primera elección en las PPHiper o PPnormC (Matthews et al. 2011; Tricarico y Camerino 2011; Cannon 2015), mientras que el mexiletine, propafenon o flecainida son los fár- macos de primera elección en el tratamiento de los síndromes miotónicos puros (Mohammadi et al. 2005; Rayan et al. 2010; Statland et al. 2012; Hahn y Salajegheh 2016).

Las canalopatías musculoesqueléticas $\mathrm{Na}_{\mathrm{v}} 1.4$ homólogas en las especies no humanas ocurren espontáneamente en caballos Cuarto de milla y sus cruzas (Rudolph et al. 1992), y han sido diseñadas genéticamente en modelos biológicos de ratones (Hayward et al. 2008; Wu et al. 2011). La mutación equina ha sido rastreada hasta su origen e identificada en un animal fundador con un fenotipo clínico de miotonía y PPHiperC; la crianza selectiva en la industria equina resultó en la rápida diseminación del defecto, que actualmente afecta al $5 \%$ de la raza (Rudolph et al. 1992). En perros se han comunicado casos con una alta presunción clínica de PPHiperC en una hembra Pit bull de 7 meses de edad (Jezyk 1982). Se ha comunicado también un caso con un video en un perro, clínicamente similar a la PMC, probablemente debido a alteraciones de los canales de sodio (Lowrie y Garosi 2017). En gatos se comunicó un cuadro clínico caracterizado por episodios de rigidez agravados por la administración de potasio, muy similar a las MAP de los humanos (Kiesewetter et al. 2011).

El objetivo de este trabajo es la comunicación de una presunta PPHiperC con miotonía en una camada de perros Shi-Tzú y Pastor Alemán, razas en las que no se ha descrito este tipo de MND. Ante la carencia de los métodos complementarios para confirmar el diagnóstico, se destaca la importancia del examen físico y neurológico, que permitió elaborar un diagnóstico presuntivo clínico-terapéutico.

\section{DESCRIPCIÓN DE LOS CASOS CLÍNICOS}

\section{Signos clínicos en perros Pastor Alemán}

Uno de los cuadros clínicos se observó en una camada de 7 cachorros Pastor Alemán (3 hembras y 4 machos) con inicio de los signos en- 
tre los 15 y los 20 días de edad. Los padres eran asintomáticos y era la primera vez que se apareaban entre ellos, aunque por separado ya lo habían hecho, sin antecedentes de enfermedad en las camadas resultantes. La familia había adquirido a la hembra a los 6 meses de edad, y al macho a los 8 meses de edad. Los cachorros nacieron en la casa, mediante parto normal. Vivían juntos en la Ciudad Autónoma de Buenos Aires, sitio en el que se realizó la interconsulta neurológica.

Los perros estaban la mayor parte del tiempo en jaulas grandes, de las cuales salían 4 veces al día. En esta situación, varios minutos después de iniciado el ejercicio, todos ellos presentaban severa rigidez muscular en los miembros pelvianos (miotonía paradójica o paramiotonía) (figura 1). La rigidez era tan intensa que provocaba la caída de los animales, que quedaban gimiendo en decúbito lateral o decúbito dorsal, sin alteraciones cognitivas (figura 2); luego de un rato lograban incorporarse y cesaba el gemido, aunque permanecían apoyados sobre el es-

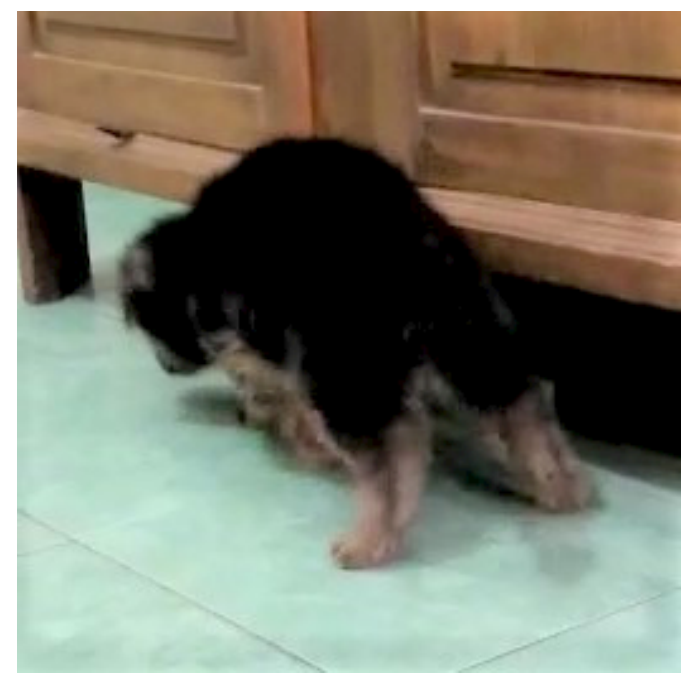

Figura 1. Varios minutos después de iniciado el ejercicio, todos los cachorros de la camada presentaban severa rigidez muscular en los miembros pelvianos (miotonía paradojal o paramiotonía). ternón y los miembros torácicos, con los miembros pelvianos rígidos en extensión, sin poder movilizarse (figura 3). La duración de los episodios era de unos segundos a pocos minutos; la frecuencia era de 4 veces en el día, siempre coincidente con la salida de la jaula para hacer ejercicio. Todos los cachorros estaban afectados, pero en los machos los signos clínicos eran más severos en intensidad, frecuencia y duración. Las manifestaciones clínicas comenzaron cuando empezaron a caminar, entre los 15 y los 20 días de vida.

Mediante la anamnesis se descartó la posibilidad de intoxicación o infección; los padres estaban correctamente vacunados y todos los cachorros estaban mamando normalmente desde su nacimiento, sin acceso a productos tóxicos.

El examen neurológico de todos los animales afectados fue normal en estado de reposo, y los signos clínicos fueron evaluados gracias a la presentación de videos caseros tomados por los propietarios. Si durante los episodios se tomaba

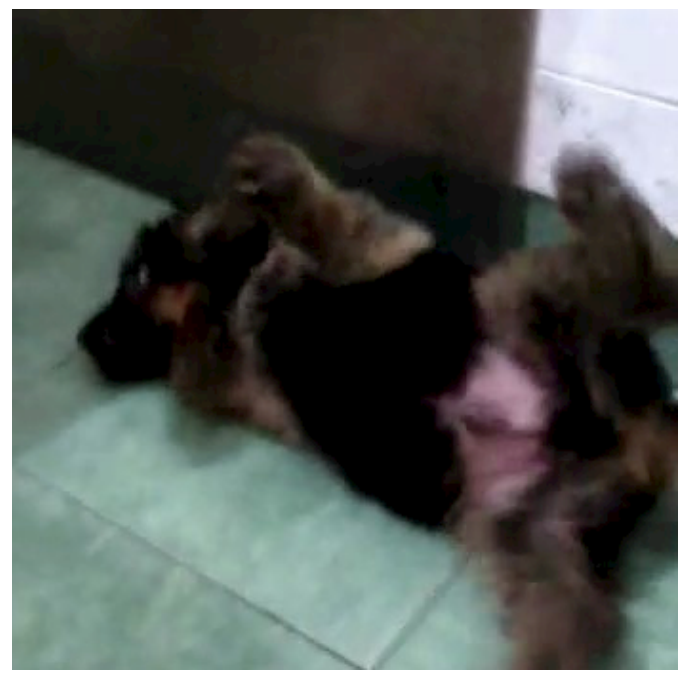

Figura 2. La rigidez era tan intensa que provocaba la caída de los animales, que quedaban gimiendo en decúbito lateral o decúbito dorsal, sin alteraciones cognitivas 


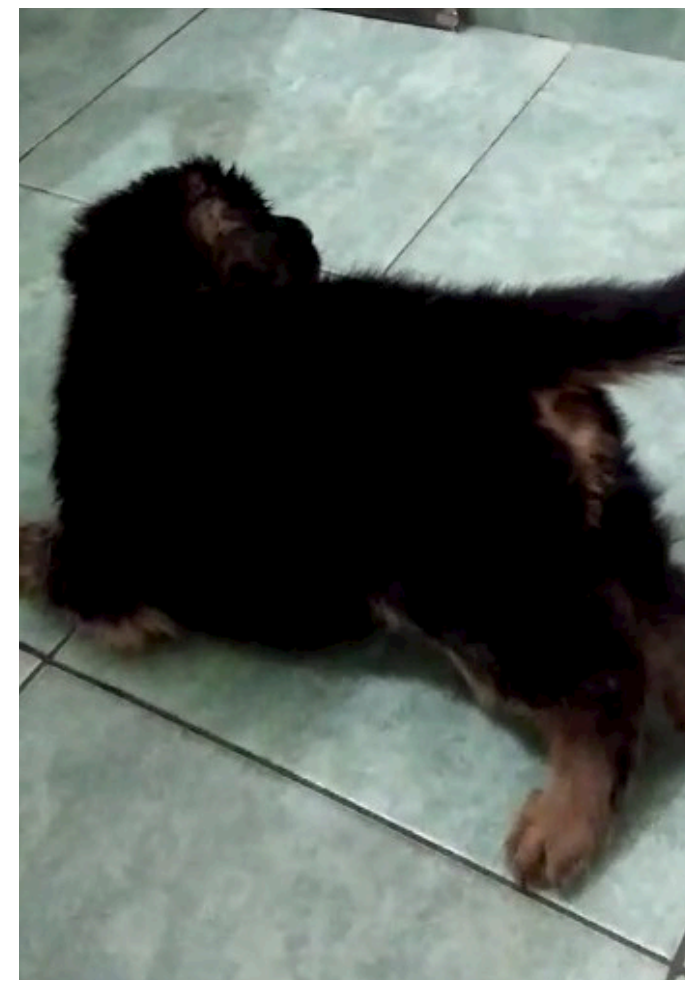

Figura 3. Luego de permanecer en decúbito lateral o dorsal unos instantes lograban incorporarse y cesaba el gemido, aunque permanecían apoyados sobre el esternón y los miembros torácicos, con los miembros pelvianos rígidos en extensión, sin poder movilizarse

en brazos a los cachorros, el gemido cesaba automáticamente; por este motivo se asumió que no se debía a la presencia de dolor, sino al miedo producido por un cuadro agudo de rigidez que imposibilitaba los movimientos con preservación del sensorio.

Ante un cuadro clínico de presentación aguda, con signos que aparecían minutos después de iniciado el ejercicio y desaparecían espontáneamente, y con un examen neurológico normal entre los episodios, los diagnósticos diferenciales que se contemplaron fueron: MND (PMC, MAP o PPHiperC con paramiotonía) o discinesia paroxística (DP), ambas con base genética y herencia autosómica. No se consideró la presencia de una miotonía congénita por alteraciones del canal de cloro porque el signo cardinal era paramiotonía, y por la ausencia del fenotipo clínico característico (por ejemplo, hipertrofia muscular en los músculos proximales). Si bien no se descartó por completo que los episodios fueran crisis epilépticas, no se consideraron entre los principales diagnósticos diferenciales por la ausencia de signos autonómicos, la conservación de la conciencia y la frecuencia de presentación sin compromiso del estado neurológico en los períodos interictales. De haber sido crisis epilépticas, las causas probables hubieran sido anomalías del desarrollo o enfermedades infecciosas; en el primer caso deberían haberse observado signos clínicos entre los episodios, y en el segundo, signos multifocales y/o sistémicos. En relación a las MND, se descartó que fuera una MAP, que se caracteriza por miotonía aislada (y no por paramiotonía).

A todos los perros se les indicó un análisis de sangre completo, que incluía hemograma, bioquímica sanguínea, ionograma y perfil tiroideo (T4 libre específica y TSH); se propuso a los propietarios la realización de un estudio electromiográfico, pero no fue aceptado. Los resultados del ionograma mostraron hipercalemia en todos los animales (entre 6.8 y 7.2 $\mathrm{mEq} / \mathrm{L}$-valores de referencia 3.7-5.2 $\mathrm{mEq} / \mathrm{L}-$ ), $\mathrm{y}$ el perfil tiroideo fue normal. En base a estos resultados se descartó la posibilidad de DP, y se indicó una terapia considerando como causal una MND (PPHiperC o PMC). Teniendo en cuenta que la $\mathrm{PMC}$ no ha sido descrita en perros (Lowrie y Garosi 2017), se inició tratamiento con acetazolamida (Diamox®, Argentina) a dosis de $10 \mathrm{mg} / \mathrm{kg}$ cada $8 \mathrm{hs}$ por vía oral. Los signos clínicos se resolvieron inmediatamente.

De acuerdo a los hallazgos clínicos y de laboratorio, y a los resultados del tratamiento con acetazolamida, el diagnóstico presuntivo fue PPHiperC con paramiotonía por una canalopatía del canal de sodio SCNAA. 
Se pudo hacer el seguimiento de la camada completa hasta 2 meses después de iniciado el tratamiento. En ese momento los cachorros permanecían asintomáticos sin cambiar la dosis de la medicación, a pesar del aumento de peso. Pasados otros 4 meses, el seguimiento se pudo hacer en 4 de los 7 perros (3 machos y 1 hembra). Todos habían abandonado la medicación entre 2 y 3 meses atrás, y ninguno de ellos repitió signos clínicos.

\section{Signos clínicos en perros Shi-Tzú}

El otro de los cuadros clínicos, observado en una camada de 4 cachorros Shi-tzú (3 hembras y 1 macho), se caracterizó clínicamente por episodios combinados de parálisis episódica y rigidez muscular, de aparición aguda, con inicio de los signos entre los 45 y los 60 días de edad. Los perros vivían en diferentes lugares (2 de ellos en la Ciudad Autónoma de Buenos Aires, uno en Pinamar, ciudad situada en la costa Atlántica de la Provincia de Buenos Aires, y el restante en la ciudad de Mendoza) y con diferentes familias, y habían sido comprados en el mismo criadero. Todos ellos fueron revisados en la Ciudad Autónoma de Buenos Aires.

Los signos clínicos fueron variando con el tiempo y entre los hermanos: en 3 de los perros consistían inicialmente en episodios de debilidad, con flacidez de los miembros pelvianos que a los pocos días se extendió a los miembros torácicos, provocando incapacidad de corregir el decúbito esternal (figura 4). Los episodios comenzaban cuando los animales, estando en reposo, iniciaban el movimiento ante la llegada de los propietarios o al comenzar a jugar; es decir, el inicio generalmente estuvo asociado a estados de excitación inmediatamente luego del reposo. La duración fluctuaba desde minutos hasta 3 horas. La frecuencia de presentación era diaria, a veces 2 o 3 episodios en el mismo día. En una de las perras, los episodios comenzaron con rigidez de los miembros pelvianos, sin flacidez (figura 5). En el transcurso de 7 a 10

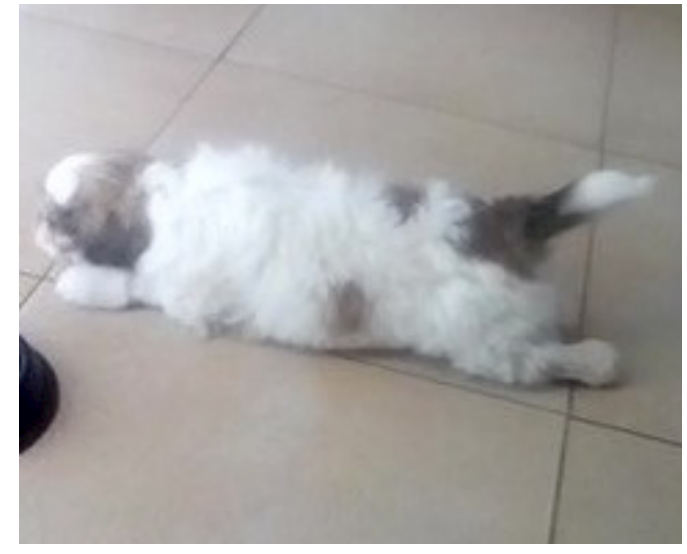

Figura 4. Episodio inicial, consistente en debilidad, con flacidez de los miembros pelvianos y los miembros torácicos, provocando incapacidad de corregir el decúbito esternal

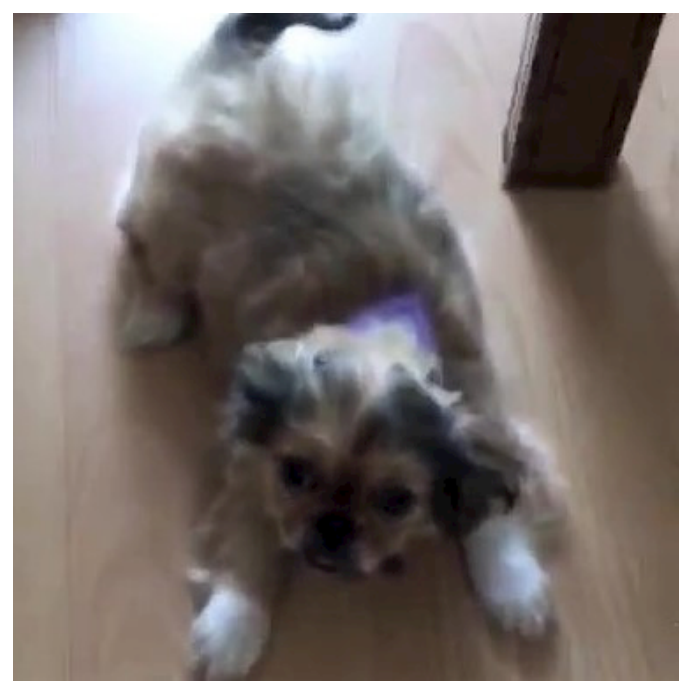

Figura 5. En una de las perras, los episodios comenzaron con rigidez de los miembros pelvianos, sin flacidez

días, todos los animales empezaron a presentar rigidez del tronco y de los miembros pelvianos; en 2 de los perros también se observaron contracciones lateralizadas de la cabeza y la musculatura cervical (figura 6), así como también el denominado signo de von Graefe o lid lag. Este 
signo es un fenómeno miotónico que consiste en la imposibilidad del párpado superior para acompañar los movimientos del globo ocular hacia abajo después de haber dirigido la mirada hacia arriba (McArdle 1962). Todos los episodios fueron notoriamente más severos en el macho que en las hembras.

Todos los animales concurrieron a la interconsulta con el servicio de neurología con análisis de sangre realizados previamente, que incluían hemograma completo, bioquímica sanguínea básica (glucemia, uremia, ALT, AST, FAS, proteínas totales, albúmina), y serología de toxoplasmosis y neosporosis. En todos los casos los resultados fueron normales, y las serologías negativas. Al macho y a una de las hembras se les había realizado previamente imágenes por resonancia magnética (IRM); en el macho se había informado el estudio como compatible con lisencefalia, aunque después de observar y analizar las imágenes se consideraron normales, al igual de las de la hembra. Ambos perros ya se encontraban medicados con anticonvulsivantes (clonazepam a dosis de 0,2 $\mathrm{mg} / \mathrm{kg}$ cada $8 \mathrm{hs}$, y fenobarbital a dosis de 2,5 $\mathrm{mg} / \mathrm{kg}$ cada $12 \mathrm{hs}$ ), sin resultados positivos en cuanto al control de los signos clínicos. Otra de las hembras ya se encontraba medicada con furosemida ( $2 \mathrm{mg} / \mathrm{kg}$ cada $12 \mathrm{hs}$ ) al momento de la interconsulta, por una presunción clínica de hidrocefalia, sin resultados positivos en lo que se refiere al control de los signos clínicos. A la hembra restante se le había realizado una tomografía computada (TC) y, con un diagnóstico presuntivo de meningoencefalitis inmunomediada, comenzó a ser medicada con prednisolona ( $1 \mathrm{mg} / \mathrm{kg}$ cada 12 hs durante una semana para continuar con $1 \mathrm{mg} / \mathrm{kg} /$ día). Los signos clínicos persistieron con la misma intensidad pero se espaciaron en el tiempo, con una frecuencia de 3 a 4 episodios por semana. Las imágenes no pudieron ser observadas, porque no se encontraban en poder del propietario.

El examen neurológico de todos los animales afectados fue normal entre los episodios, $\mathrm{y}$

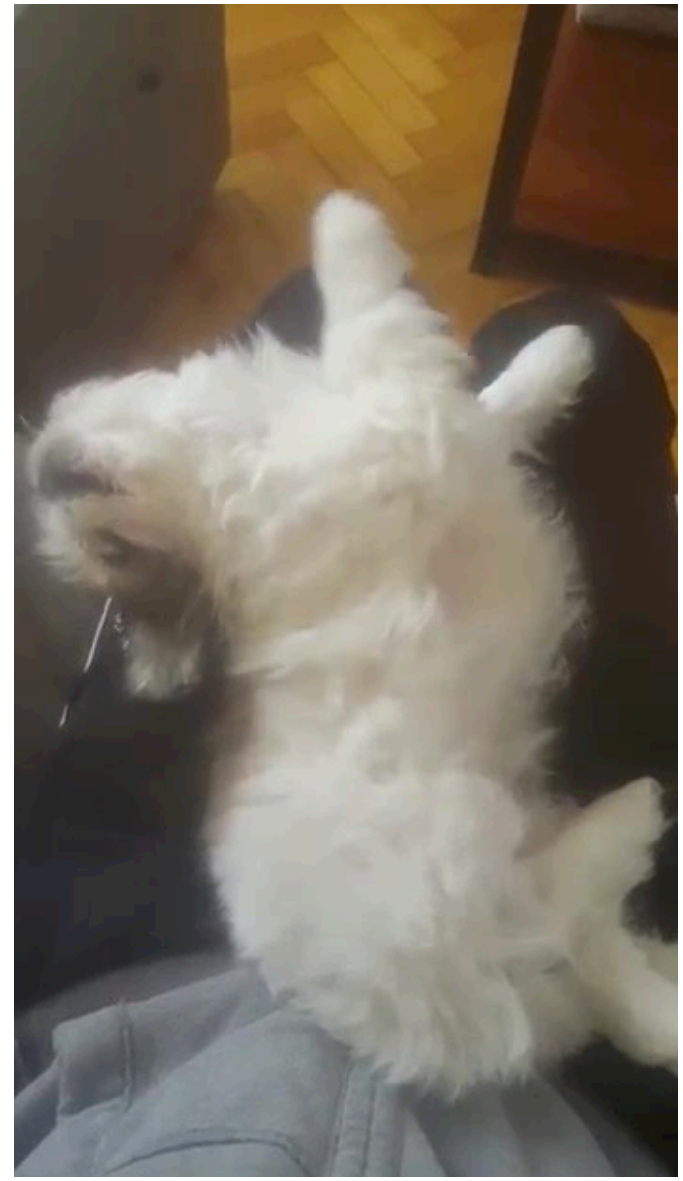

Figura 6. A partir de la semana de iniciado el cuadro clínico, los episodios consistían en rigidez del tronco y de los miembros pelvianos; en 2 de los perros también se observaron contracciones lateralizadas de la cabeza y la musculatura cervical

por ese motivo se desestimaron los diagnósticos que consideraban trastornos estructurales (lisencefalia, hidrocefalia) o inflamatorios (meningoencefalitis inmunomediada). Los signos clínicos fueron evaluados gracias a la presentación de videos caseros tomados por los propietarios.

Ante un cuadro clínico de presentación aguda, con signos que aparecían y desaparecían espontáneamente y examen neurológico normal 


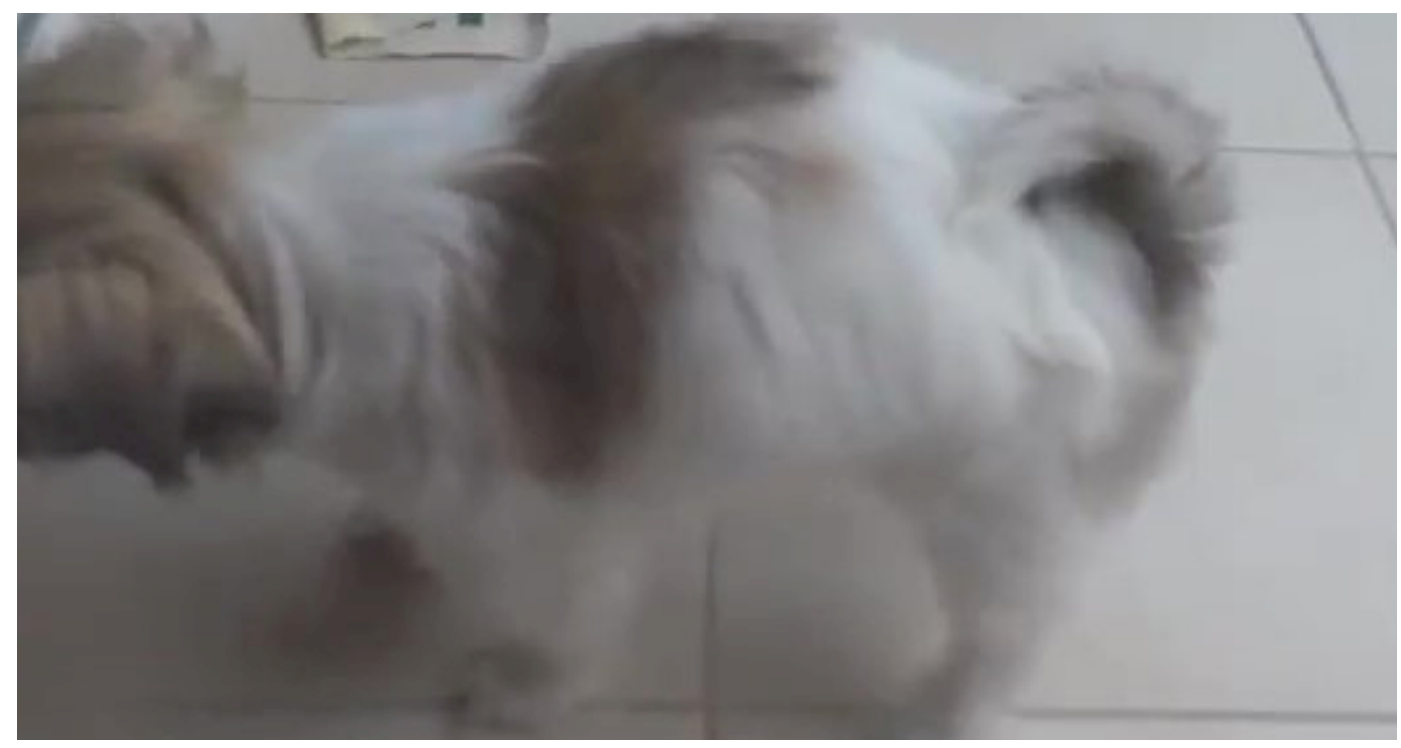

Figura 7. En los primeros 2 meses de tratamiento los signos clínicos se limitaron a episodios de rigidez de ambos miembros pelvianos ("salto de conejo") al iniciar la marcha o la carrera luego del reposo, desencadenados generalmente por situaciones de excitación

entre los episodios, los diagnósticos diferenciales que se contemplaron fueron: crisis epilépticas; DP; MND. Por la gran duración de las crisis (hasta 3 horas), la ausencia de signos autonómicos y la falta de respuesta al tratamiento anticonvulsivante en los 2 perros en los que se había implementado, se descartó que los signos correspondieran a crisis epilépticas; la duración solamente se hubiera explicado por la presencia de lesiones estructurales, que se habían descartado mediante IRM en 2 de los perros. Por la raza afectada, que en nuestro conocimiento no padece de ninguna DP comunicada, y por la duración de los ataques se consideró que este tipo de trastorno era muy poco probable. En consecuencia, el diagnóstico presuntivo a partir del que se elaboró la estrategia diagnóstica fue el de MND. Como ninguno de los animales afectados presentaba signos permanentes que permitieran sospechar patología muscular obvia, tales como marcha rígida, hipometría, dolor muscular, hipertrofia muscular o signo del hoyuelo, se consideró una MND por alteración del canal de sodio. Debido a la relación de parentesco entre los animales afectados se sospechó una base genética, aunque no se pudieron averiguar antecedentes confiables en relación a los padres o a otras camadas.

En base a esta presunción diagnóstica, a todos los perros se les indicó un ionograma completo y un perfil tiroideo (T4 libre específica y TSH); se propuso a los propietarios la realización de un estudio electromiográfico, pero no fue aceptado. A uno de los perros (al macho) se le indicó potasio por vía oral como prueba diagnóstica. Los resultados del ionograma mostraron hipercalemia en una de las hembras (6.5 $\mathrm{mEq} / \mathrm{L}$-valores de referencia 3.7-5.2 $\mathrm{mEq} / \mathrm{L}-$ ), $\mathrm{y}$ el perfil tiroideo fue normal en los 4 animales. En el perro al que se le suministró potasio se observó un empeoramiento de los signos clínicos, en relación a su frecuencia e intensidad.

De acuerdo a los hallazgos clínicos y de laboratorio, el diagnóstico presuntivo fue PPHiperC con miotonía debido a una canalopatía del canal de sodio (Jurkat-Rott et al. 2002; 2015; 
Jurkat-Rott y Lehmann-Horn 2010). Se inició un tratamiento con acetazolamida (Diamox $®$, Argentina) a dosis de $10 \mathrm{mg} / \mathrm{kg}$ cada $8 \mathrm{hs}$.

Con la administración de acetazolamida las manifestaciones clínicas mejoraron inicialmente (luego de 3 o 4 días); la mejoría se manifestó en la frecuencia de los episodios, que pasaron a ser semanales o bisemanales, y en su intensidad. En los primeros 2 meses las manifestaciones clínicas se limitaron a episodios de rigidez de ambos miembros pelvianos ("salto de conejo") al iniciar la marcha o la carrera luego del reposo, desencadenados generalmente por situaciones de excitación (figura 7), para desaparecer por completo luego de 3 meses de tratamiento, sin cambiar la dosis de la medicación. La acetazolamida fue discontinuada entre $\operatorname{los} 7$ y los 8 meses del diagnóstico, en forma gradual. En la actualidad, a 9 meses de haber realizado el diagnóstico presuntivo y entre 1-2 meses de haber abandonado el tratamiento, los cachorros se encuentran completamente asintomáticos.

\section{DISCUSIÓN}

Los casos clínicos descritos en este trabajo se asemejan a la PPHiperC con miotonía/paramiotonía de los humanos (Jurkat-Rott et al. 2002, 2015; Jurkat-Rott y Lehmann-Horn 2010; Okuda et al. 2001; Wagner et al. 1997).

Los hechos que sustentaron el diagnóstico presuntivo fueron la localización neuroanatómica de los signos clínicos (sistema muscular), la edad de presentación, la relación de parentesco entre los animales afectados, el desencadenamiento de los signos clínicos (debilidad y miotonía) en relación al ejercicio (al inicio en los perros Shi-Tzú, luego de un rato de iniciado en los perros Pastor Alemán), la duración de los ataques, la presencia de hipercalemia en todos los perros Pastor Alemán y en uno de los de raza Shi-Tzú, el empeoramiento de los signos clínicos con la administración de potasio en uno de los perros Shi-Tzú y la resolución de los signos luego de administrarles acetazolamida en todos los perros (Camacho 1984; Okuda et al. 2001; Jurkat-Rott et al. 2002, 2015; Jurkat-Rott y Lehmann-Horn 2010; Kim 2014; Statland et al. 2018).

En la camada de perros Pastor Alemán, la diferenciación entre PMC y PPHiperC con paramiotonía fue dificultosa debido a que los signos clínicos se superponen entre ambos trastornos (Statland et al. 2018); se realizó en base a la duración de los episodios, a los hallazgos de laboratorio y a la respuesta a la terapia con un inhibidor de la anhidrasa carbónica. Previamente a la disponibilidad de pruebas de genética molecular existían argumentos para afirmar que ambos trastornos eran una misma enfermedad nosológica. Inclusive, en algunas familias humanas, los pacientes con PMC desde el nacimiento muestran episodios de PPHiperC en la adolescencia (Rayan et al. 2010; Kumar et al. 2014; Hahn y Salajegheh 2016). La diferenciación entre ambas patologías es importante porque el tratamiento es diferente; en PMC las drogas de primera elección son el mexiletine, propafenon o flecainida (Mohammadi et al. 2005; Rayan et al. 2010; Statland et al. 2012; Hahn y Salajegheh 2016), mientras que en PPHiperC son los inhibidores de la anhidrasa carbónica (Matthews et al. 2011; Tricarico y Camerino 2011; Cannon 2015). La excelente respuesta de los cachorros afectados a la acetazolamida fue uno de los elementos que nos permitieron diferenciar entre PPHiperC y PMC.

El tratamiento de la PPHiperC consiste en disminuir la hipercalemia con fármacos como la acetazolamida, mineralocorticoides, fludrocortisona y dietas bajas en potasio (Naberhaus et al. 2008; Jurkat-Rott y Lehmann-Horn 2010; Zapata-Wainberg et al. 2015; Dewey y Talarico 2016). En todos los casos presentados en este trabajo la respuesta a la acetazolamida fue exitosa; en los perros Pastor Alemán la resolución de los signos fue inmediata. En los perros ShiTzú todos los animales respondieron satisfactoriamente a la administración de acetazolamida, atenuándose los signos clínicos al inicio de la 
terapia, para desaparecer por completo luego de 3 meses de tratamiento. Se supone que la acetazolamida estimula la liberación tanto de insulina como de glucagón, lo que promueve en forma subsecuente el influjo de potasio hacia el interior de la célula muscular, mejorando los signos clínicos de la PPHiperC (Dewey y Talarico 2016). La mejoría parcial de uno de los perros Shi-Tzú tratado con prednisolona fue atribuida a la acción de los glucocorticoides sobre el balance electrolítico, incrementando la eliminación de potasio en el tracto intestinal (Cosío et al. 2005).

Llama la atención el hecho de no haber tenido que ajustar la dosis con el aumento de peso de los animales debido al crecimiento y también que, al retirar la medicación, no hubo recidivas. Podría deberse a que la enfermedad sea autolimitante en el tiempo, tal como sucede en los humanos con PPhiperC, en los que se observa una disminución de la frecuencia y gravedad de los signos clínicos a partir de los 40 años (Platt y Griggs 2009; Zapata-Wainberg et al. 2015). La remisión también podría resultar a partir de una sensibilidad al tiempo de la expresión del gen mutado, tal como sucede en algunas epilepsias juveniles con remisión espontánea (por ej., epilepsia rolándica benigna o epilepsia de ausencia infantil en los humanos, o epilepsia benigna juvenil familiar benigna del Lagotto Romagnolo en los perros) (Roger et al. 2005; Jokinen et al. 2007; Seppala et al. 2011; 2012). En estos casos, los signos comienzan en la segunda fase del desarrollo cerebral pos natal, durante el cual se expresan genes mutados que son reemplazados en la fase final por genes homólogos normales (Arsenault y Zhang 2006).

Los niveles de potasio sérico estuvieron francamente elevados en todos los perros Pastor Alemán; en cambio, los niveles obtenidos de los 4 cachorros Shi-Tzú demostraron la presencia de hipercalemia en uno solo de ellos, resultando normocalémicos los demás. La hipercalemia es muy rara en PMC, siendo la mayoría de los pacientes afectados nor- mocalémicos (Zapata-Wainberg et al. 2015). La normocalemia no descarta la presencia de PPHiperC; muchas personas con este tipo de MND son normocalémicas durante los ataques (Sansone et al. 2012; Jurkat-Rott et al. 2015; Zapata-Wainberg 2015; Statland et al. 2018). En una larga serie de pacientes humanos con la mutación T704M SCN4A (una de las 2 más frecuentes en PPHiperC) la hipercalemia durante las crisis se observó solamente en el 50\% de los casos (Plassart et al. 1994). En una época se consideró a la PPnormoC como un tercer tipo de PP, generando un debate acerca de su real existencia (Poskanzer y Kerr 1961). Un análisis retrospectivo clínico y molecular en familias con PPnormoC demostró mutaciones T704M o M1592V SCN4A, que previamente habían sido descritas como causales de PPHiperC, confirmando que la PPnormoC es simplemente una variante fenotípica de la PPHiperC, y no una enfermedad distinta (Chinnery et al. 2002).

En relación a la duración de los episodios, en PPHiperC son relativamente breves, entre 15 minutos y 4 horas (Rose y Griggs 2009), mientras que en PMC suelen durar de horas a varios días (Zapata-Wainberg et al. 2015). En los casos presentados en este trabajo la duración nunca excedió de unos pocos minutos para los perros Pastor Alemán, ni las 3 horas para los perros Shi-Tzú.

Debido a la relación de parentesco y la presentación simultánea de los signos clínicos en todos los perros, se sospechó una base genética con herencia autosómica. En los humanos, la PPHiperC es de transmisión autosómico dominante. Si sucediera lo mismo en los perros, al menos uno de los padres en ambas camadas debería haber presentado signos clínicos. En el caso de los perros Pastor Alemán, la ausencia de signos clínicos en los padres podría deberse al desconocimiento de la historia en los primeros meses de vida (fueron adquiridos a los 6 y 8 meses de edad), o a que la penetrancia de la enfermedad en esta especie fuera reducida, como sucede con algunas mutaciones puntuales 
que provocan PPHiperC con paramiotonía en los humanos (McClatchey et al. 1992; Wagner et al. 1997). En el caso de los perros Shi-Tzú, por desgracia no se pudieron obtener datos confiables acerca de los padres por falta de colaboración de los criadores. Con respecto al carácter autosómico, en ambas camadas enfermaron tanto las hembras y los machos, aunque en estos últimos los signos clínicos fueron mucho más severos. En medicina humana se describieron casos de mutaciones puntuales en el gen SCN4A que causan PPHiperC con paramiotonía en los varones, y solamente descargas miotónicas visibles en el electromiograma en las mujeres, lo que sugiere modificaciones en la expresión clínica relacionadas al sexo. Los autores propusieron una expresividad clínica inferior en las mujeres, causada por una dependencia de la excitabilidad de la fibra muscular en las hormonas sexuales, o la expresión de un canal adicional, con participación en la estabilización del sarcolema, codificado solamente por el cromosoma X (Wagner et al. 1997).

No se pudieron realizar pruebas genéticas para identificar la mutación SCN4A. En medicina humana, en ausencia de la identificación de la mutación genética, la PPHiperC puede diagnosticarse en base a la presentación clínica, los niveles de potasio sérico durante la crisis y los patrones de anormalidad durante un ejercicio prolongado. Si se sospecha de PPHiperC primaria debe confirmarse que los signos no son secundarios a otras condiciones como tirotoxicosis (Lin 2005) u otras causas que provoquen discalemias (Statland et al. 2018). Los perros en cuestión eran eutiroideos y sin otras patologías sistémicas que pudieran afectar las concentraciones séricas de potasio.

La presentación clínica de las MND es compleja en todas las especies, y a menudo variable. Aunque algunos métodos de diagnóstico complementario pueden proveer la confirmación de muchos de estos trastornos, o al menos descartar enfermedades que las mimetizan, su valor es limitado debido al origen funcional de estas condiciones (Abdo et al. 2010; Forman et al. 2012). En consecuencia, se enfatiza en el hecho que el diagnóstico se apoya fundamentalmente en las observaciones surgidas del examen clínico (Abdo et al. 2010; Lowrie y Garosi 2017), tal como lo hemos hecho en los casos descritos.

En medicina veterinaria, la MND por alteración en los canales de sodio se ha demostrado y caracterizado en caballos Cuarto de milla y sus mestizos (Spier et al. 1990; Rudolph et al. 1992; Meyer et al. 1999), y se ha comunicado su fuerte sospecha en perros (Jezyk 1982); ha sido denominado PPHiperC (Lowrie y Garosi 2017).

En los caballos Cuarto de milla se ha identificado la mutación responsable del trastorno, que consiste en un cambio de fenilalanina a leucina en uno de los dominios transmembrana de la proteína SCN4A homóloga a la humana (Rudolph et al. 1992). El perfil de la enfermedad es muy similar en todos los aspectos a la forma humana de PPHiperC, con 2 excepciones: en caballos siempre cursa con miotonía, y se han descrito casos de muerte súbita durante los episodios (Meyer et al. 1999); en la actualidad constituye un modelo animal espontáneo para esta enfermedad (Naberhaus et al. 2008).

En los perros, se comunicó la sospecha de PPHiperC en una hembra Pit bull de 7 meses de edad que presentaba episodios de 10 a 15 segundos de colapso asociado al ejercicio, hipotonía en los miembros y en el cuello, y protrusión de la lengua. Los episodios se hicieron más frecuentes con el correr del tiempo. El diagnóstico se apoyó en los signos clínicos, sustentado por un aumento de las concentraciones séricas de potasio. Los signos clínicos se exacerbaron con la administración de potasio por vía oral, lo que se utilizó como apoyo diagnóstico (Jezyk 1982).

Un caso de DP responsiva a la acetazolamida fue comunicado en una hembra de raza Retriever Dorado de 12 semanas de edad. La mayoría de los episodios se desencadenaban por excitación y ejercicio y comenzaban con 
marcha rígida y cifosis, progresando a descenso de la cabeza y elevación de los miembros pelvianos. Finalmente, la perra colapsaba con rigidez extensora en los 4 miembros. Una vez desaparecido el factor desencadenante, el tono muscular volvía a la normalidad y los signos desaparecían. La frecuencia de los episodios era de 1 a 8 por día, y la duración variaba de unos pocos segundos a un máximo de 10 minutos. Entre los episodios la perra era completamente normal, y los resultados del hemograma, la bioquímica sanguínea y los electrólitos fueron normales. Los signos desaparecieron completamente 3 días después de comenzar la terapia con acetazolamida (Royaux et al. 2016). Este caso difiere a los que presentamos en este trabajo en la ausencia de episodios de flacidez, y en la normalidad de los análisis de laboratorio.

En 12 gatos Europeos de pelo corto se comunicó un cuadro de rigidez muscular agravada por potasio. La edad de inicio de los signos clínicos varió entre los 2 meses a los 3 años y, aunque se sospechó una relación genética entre los animales afectados, no pudo comprobarse. Los resultados de la evaluación neurológica fueron normales en los gatos durante el reposo, pero todos ellos manifestaron episodios de espasticidad muscular inducidos por el estrés o por el ejercicio. Los exámenes complementarios no evidenciaron ningún tipo de anormalidad; sin embargo, la administración de una dieta enriquecida con potasio resultó en severo agravamiento de los signos clínicos, lo que permitió el diagnóstico de rigidez muscular agravada por potasio (Kiesewetter et al. 2011).

\section{CONCLUSIONES}

En base a la fenomenología clínica (debilidad con paramiotonía en los perros Pastor Alemán, debilidad con flacidez y miotonía en los perros Shi-Tzú, edad de presentación, parentesco entre los animales afectados, desencadenamiento de los signos clínicos en relación al ejercicio, presencia de hipercalemia en todos los individuos de raza Pastor Alemán y en uno de los perros Shi-Tzú), la ocurrencia episódica, la ausencia de signos clínicos entre los episodios y la duración de los mismos, y su empeoramiento cuando se administró potasio por vía oral en uno de los perros Shi-Tzú se propone a esta descripción como la primera comunicación de PPHiperC con paramiotonía en perros Pastor Alemán y PPHiperC con miotonía en perros Shi-Tzú. Tal diagnóstico presuntivo está apoyado por la respuesta favorable tras el inicio de la terapia con acetazolamida. Se propone incorporar estas razas al grupo de perros afectados por PPHiperC con miotonía. El curso de la enfermedad en todos los perros observados indica un curso regresivo, con un pronóstico favorable.

\section{AGRADECIMIENTOS}

A la Dra. Sofia Cerda-Gonzalez, y a los Dres. Ronaldo Casimiro da Costa y Martí Pumarola Batlle por sus sugerencias y comentarios, que contribuyeron a mejorar el artículo.

\section{REFERENCIAS BIBLIOGRÁFICAS}

1. Abdo, W.F., van de Warrenburg, B.P., Burn, D.J., et al. (2010). The clinical approach to movement disorders, Nat Rev Neurol, 6, 2937.

2. Arsenault D., Zhang, Z.W. (2006) Developmental remodelling of the lemniscal synapse in the ventral basal thalamus of the mouse, J Physiol 573(Pt 1),121-132.

3. Brancati, F., Valente, E.M., Davies, N.P., Sarkozy, A., Sweeney, M.G., Lo-Monaco, M., et al. (2003). Severe infantile hyperkalaemic periodic paralysis and paramyotonia congenita: broadening the clinical spectrum associated with the T704M mutation in SCN4A, J Neurol Neurosurg Psychiatry;74:1339-41.

4. Camacho, L.M. (1984). Parálisis periódica paramiotónica. Estudio de una familia colombiana, Acta Méd Col, 9(2), 53-59. 
5. Cannon, SC. (2002). An expanding view for the molecular basis of familial periodic paralysis, Neuromusc Disord,12, 533-543.

6. Cannon, S.C. (2015). Channelopathies of skeletal muscle excitability, Compr Physiol, 5, 761-790.

7. Cannon, S.C. (2018). Sodium channelopathies of skeletal muscle, Handb Exp Pharmacol, 246, 309-330.

8. Chinnery, P.F., Walls, T.J., Hanna, M.G., Bates, D., Fawcett, P.R. (2002). Normokalemic periodic paralysis revisited: does it exist? Ann Neurol, 52(2), 251-252.

9. Cosío, B.G., Torrego, A., Adcock, I.M. (2005) Mecanismos moleculares de los glucocorticoides, Arch Bronconeumol, 41, 34-41

10. Dewey, C.W., Talarico, L.R. (2016). Myopathies: disorders of skeletal muscle (chapter 18). En: C.W. Dewey, R.C. da Costa (Ed.), Practical guide to canine and feline neurology. $3^{\text {rd }}$ ed. (pp 481-520). New Delhi, India:Wiley Blacwell.

11. Forman, O.P., Penderis, J., Hartley, C., Hayward, L.J., Ricketts, S.L., Mellersh, C.S. (2012). Parallel mapping and simultaneous sequencing reveals deletions in BCAN and FAM83H associated with discrete inherited disorders in a domestic dog breed, PLoS Genetics, 8, e1002462.

12. George, A.L. Jr, Komisarof , R.G., Barchi, R.L. (1992). Primary structure of the adult human skeletal muscle voltage-dependent sodium channel, Annals of Neurology, 31, 131-137.

13.Hahn, C., Salajegheh, M.K. (2016). Myotonic disorders: a review article, Iran J Neurol, 15(1), 46-53.

14. Hayward, L.J., Kim, J.S., Lee, M.Y., Zhou, H., Kim, J.W., Misra, K., Salajegheh, M., Wu, F.F., Matsuda, C., Reid, V., Cros, D., Hoffman, E.P., Renaud, J.M., Cannon, S.C., Brown, R.H. (2008). Targeted mutation of mouse skeletal muscle sodium channel produces myotonia and potassium-sensitive weakness, J Clin Invest, 118, 1437-1449.
15. Huang, W., Liu, M., Yan, S.F., Yan, N. (2017). Structure-based assessment of disease-related mutations in human voltagegated sodium channels, Protein Cell, 8, 401-438.

16. Isom, L.L., De Jongh, K.S., Patton, D.E., Reber, B.F., Offord, J., Charbonneau, H., Walsh, K., Goldin, A.L., Catterall, W.A. (1992). Primary structure and functional expression of the beta 1 subunit of the rat brain sodium channel, Science, 256, 839-842.

17. Jezyk, P.F. (1982). Hiperkalemic periodic paralysis in a dog, J Am Anim Hosp Assoc, 18, 977-980.

18. Jokinen, T.S., Metsahonkala, L., Bergamasco, L., Viitmaa, R., Syrja, P., et al. (2007) Benign familial juvenile epilepsy in lagotto romagnolo dogs, J Vet Intern Med, 21(3), 464-471.

19. Jurkat-Rott, K., Mitrovic, N., Hang, C., et al. (2000). Voltage-sensor sodium channel mutations cause hypokalemic periodic paralysis type 2 by enhanced inactivation and reduced current, Proc Natl Acad Sci USA, 97, 9549-9554.

20. Jurkat-Rott, K., Lerche, H., Lehmann-Horn, F. (2002). Skeletal muscle channelopathies, J Neurol, 249, 1493-1502.

21. Jurkat-Rott, K., Lehmann-Horn, F. (2010). State of the art in hereditary muscle channelopathies, Acta Myologica, XXIX, 343-350.

22. Jurkat-Rott, K., Rüdel, R., Lehmann-Horn, F. (2015). Muscle channelopaties: myotonias and periodic paralyses (chapter 38). En: B.T. Darras, H.R. Jones, M.M. Ryan, D.C. De Vivo (Ed.). Neuromuscular disorders of infancy, childhood, and adolescence (2nd ed.) (pp 719-734). China:Elsevier Inc.

23. Kiesewetter, I.S., Tipold, A., Baumgärtner, W., Schenk, H.C. (2011). Potassium-aggravated muscle stiffness in 12 cats, $\mathrm{J}$ Am Vet Med Assoc, 238(8), 1026-1031.

24. Kim J., Hahn, Y., Sohn, H., Lee, Y.J., Yun, J.H., Kim, J.M., et al. (2001). Phenotypic variation of a Thr704Met mutation in ske- 
letal sodium channel gene in a family with paralysis periodica paramyotonica, $\mathrm{J}$ Neurol Neurosurg Psychiatry, 70, 618-23.

25. Kim, J. (2014). Channelopaties, Korean J Pediatr, 57(1), 1-18.

26.Kumar, D., Ambasta, R.K., Kumar, P. (2014). Mutational consequences of aberrant ion channels in meurological disorders, J Membrane Biol, 247, 1083-1127.

27. Lehmann-Horn, F., Rüdel, R., JurkatRott, K. (2004). Nondystrophic myotonias and periodic paralyses (chapter 46). En: A.G. Engel, C. Franzini-Armstrong (Ed.). Myology. 3rd ed. (pp 1257-1300). New York:McGraw-Hill.

28. Lin, S.H. (2005). Thyrotoxic periodic paralysis, Mayo Clin Proc, 80, 99-105

29. Lowrie, M., Garosi, L. (2017). Classification of involuntary movements in dogs: myoclonus and myotonia, J Vet Intern Med, 31, 979-987.

30. Matthews, E., Portaro, S., Ke, Q., Sud, R., Hawort,h A., Davis, M.B., et al. (2011). Acetazolamide efficacy in hypokalemic periodic paralysis and the predictive role of genotype, Neurology, 77, 1960-1964.

31. McArdle, B. (1962). Adynamia episodica hereditaria and its treatment, Brain, 85, 12148.

32. McClatchey, A.I., McKenna-Yasek, D., Cros, D., Worthen, H.G., Kuncl, R.W., DeSilva, S.M., Cornblath, D.R., Gusella, J.F., Brown, R.H. Jr. (1992). Novel mutations in families with unusual and variable disorders of the skeletal muscle sodium channel, Nat Gen, 2, 148-152.

33. Meyer, T.S., Fedde, M.R., Cox, J.H., Erickson, H.H. (1999). Hyperkalaemic periodic paralysis in horses: a review, Equine Vet $\mathrm{J}$, 31, 362-7.

34. Miller, T.M., Dias da Silva, M.R., Miller, H.A., Kwiecinski, H., Mendell, J.R., Tawil, R., McManis, P., Griggs, R.C., Angelini, C., Servidei, S., Petajan, J., Dalakas, M.C., Ranum, L.P., Fu Y.H., Ptacek, L.J. (2004). Co- rrelating phenotype and genotype in the periodic paralyses, Neurology, 63, 1647-1655.

35. Mohammadi, B., Jurkat-Rott, K., Alekov, A. (2005). Preferred mexiletine block of human sodium channels with IVS4 mutations and its $\mathrm{pH}$ dependence, Pharm Gen, 15, 235-44

36. Naberhaus, B., Cormand, B., Cuenca-León, E., Ribasés, M., Monells, J. (2008). Parálisis periódica hipercaliémica: presentación de una familia española con la mutación p.Thr704Met en el gen $S C N 4 A$, Neurología, 23(7), 427-435.

37. Okuda, S., Kanda, F., Nishimoto, K., Sasaki, R., Chihara, K. (2001). Hyperkalemic periodic paralysis and paramyotonia congenita. A novel sodium channel mutation, J Neurol, 249, 1003-4.

38. Plassart, E., Reboul, J., Rime, C.S., et al. (1994). Mutations in the muscle sodium channel gene (SCN4A) in 13 French families with hyperkalemic periodic paralysis and paramyotonia congenita: phenotype to genotype correlations and demonstration of the predominance of two mutations, Eur $\mathbf{J}$ Hum Genet, 2, 110-124.

39. Platt, D., Griggs, R. (2009). Skeletal muscle channelopathies: new insights into the periodic paralyses and nondystrophic myotonias, Curr Opin Neurol, 22(5), 524-31.

40. Poskanzer, D.C., Kerr, D.N. (1961). A third type of periodic paralysis, with normokalemia and favourable response to sodium chloride, Am J Med Sep, 31, 328-342.

41. Ptacek, J.L., Tyler, F., Trimmer, J.S., Agnew, W.S., Leppert ,M. (1991a). Analysis in a large hyperkalemic periodic paralysis pedigree supports tight linkage to a sodium channel locus, Am J Hum Genet, 49, 37882.

42. Ptacek, L.J., George, A.L. Jr, Griggs, R.C., Tawil, R., Kallen, R.G., Barchi, R.L., et al. (1991b). Identification of a mutation in the gene causing hypercalemic periodic paralysis, Cell, 67, 1021-7. 
43.Ptacek, L.J., Kohnson, K.J., Griggs, R.C. (1993). Genetics and physiology of the myotonic muscle disorders, N Eng J Med, 328(7), 482-489.

44. Rayan, R., Dipa, L., Michael, H.G. (2010). Skeletal muscle channelopathies: nondystrophic myotonias and periodic paralysis, Current Opinion in Neurology, 23(5), 46676.

45.Renner, D.R., Ptacek, L.J. (2001). Periodic paralyses and nondystrophic myotonias, Neuromusc Disord, 15, 235-52.

46. Ricker, K., Rohkamm, R., Böhlen, R. (1986). Adynamia episodica and paralysis periodica paramyotonica, Neurology, 36, 682-6.

47.Roger, J., Bureau, M., Dravet, C., Genton, P., Tassinari, C.A., et al. (2005) Epileptic syndromes in infancy, childhood and adolescence (4th edition). John Libbey Eurotext. $616 \mathrm{p}$.

48.Rojas, C.V., Wang, J., Schwartz, L.S., Hoffman, E.P., Powell, B.R., Brown, R.H. (1991). A Met-to-Val mutation in the skeletal muscle $\mathrm{Na}^{+}$channel alfa-subunit, Nature, 354, 387-9.

49. Rose, M., Griggs, R.C. (2009). Hereditary nondegenerative neuromuscular disease. En: C.G. Goetz (Ed). Textbook of clinical neurology. $3^{\text {a }}$ ed. (pp. 813-826). Philadelphia: Saunders.

50. Royaux, E., Bhatti, S., Harvey, R. et al. (2016) Acetazolamide-responsive paroxysmal dyskinesia in a 12-week-old female golden retriever dog, Vet Quart, 36, 45-49.

51. Rüdel, R., Lehmann-Horn, F. (1985). Membrane changes in cells from myotonia patients, Physiol Rev, 65, 310-356.

52.Rüdel, R., Ricker, K., Lehmann-Horn, F. (1993). Genotype-phenotype correlations in human skeletal muscle sodium channel diseases, Arch Neurol, 50, 1241-1248.

53. Rudolph, J.A., Spier, S.J., Byrns, G., Rojas, C.V., Bernoco, D., Hoffman, E.P. (1992). Periodic paralysis in quarter horses: a so- dium channel mutation disseminated by selective breeding, Nat Gen, 2, 144-147.

54. Sansone, V.A., Ricci, C., Montanari, M., Apolone, G., Rose, M., Meola, G.; INQoL Group. (2012). Measuring quality of life impairment in skeletal muscle channelopathies, Eur J Neurol, 19, 1470-1476.

55. Seppala, E.H., Jokinen, T.S., Fukata, M., Fukata, Y., Webster, M.T., et al. (2011) LGI2 truncation causes a remitting focal epilepsy in dogs, PLoS Genet, 7(7), e1002194. doi: 10.1371/journal.pgen.1002194.

56. Seppala, E.H., Koskinen, L.L.E., Gulløv, C.H., Jokinen, P., Karlskov-Mortensen, P., et al. (2012) Identification of a Novel Idiopathic Epilepsy Locus in Belgian Shepherd Dogs,, PLoS ONE, 7(3): e33549. doi:10.1371/journal.pone.0033549.

57.Spier, S.J., Carlson, G.P., Holliday, T.A., Cardinet, G.H. 3rd, Pickar, J.G. (1990). Hiperkalaemic periodic paralysis in horses, $\mathrm{J}$ Am Vet Med Assoc, , 1971009-1017.

58. Spillane, J., Kullmann, D.M., Hanna, M.G. (2016). Genetic neurological channelopathies: molecular genetics and clinical phenotypes, J Neurol Neurosurg Psychiatry, 87, 37-48.

59. Statland, J.M., Bundy, B.N., Wang, Y., Rayan, D.R., Trivedi, J.R., Sansone, V.A., et al. (2012). Mexiletine for symptoms and signs of myotonia in nondystrophic myotonia: a randomized controlled trial, JAMA, 308(13), 1357-65.

60. Statland, J.M., Fontaine, B., Hanna, M.G., Johnson, K.E., Kissel, J.T., et al. (2018). Review of the diagnosis and treatment of periodic paralysis, Muscle Nerve, 57, 522530 .

61. Sternberg, D., Maisonobe, T., Jurkat-Rott, K., et al. (2001). Hypokalaemic periodic paralysis type 2 caused by mutations at codon 672 in the muscle sodium channel gene SCN4A, Brain, 124, 1091-1099.

62. Tricarico, D., Camerino, D.C. (2011). Recent advances in the pathogenesis and drug 
action in periodic paralyses and related channelopathies, Front Pharmacol 2, 8.

63. Trimmer, J.S., Cooperman, S.S., Tomiko, S.A., Zhou, J., Crean, S.M., Boyle, M.B., Kallen, R.G., Sheng, Z., Barchi, R.L., Sigworth, F.J., Goodman, R.H., Agnew, W.S., Mandel, G. (1989). Primary structure and functional expression of a mammalian skeletal muscle sodium channel, Neuron, 3, 33-49.

64. Vicart, S. (2004). New mutations of SCN4A cause a potassium-sensitive normokalemic periodic paralysis, Neurology, 63, 2120-7.

65. Wagner, S., Lerche, H., Mitrovic, N., et al. (1997) A novel sodium channel mutation causing a hyperkalemic paralytic and paramyotonic syndrome with variable clinical expressivity, Neurology, 49, 1018-1025

66. Wu, F., Mi, W., Burns, D.K., Fu, Y., Gray, H.F., Struyk, A.F., Cannon, S.C. (2011). A sodium channel knockin mutant (NaV1.4$\mathrm{R} 669 \mathrm{H})$ mouse model of hypokalemic periodic paralysis, J Clin Invest, 121, 4082- 4094.
67.Zaharieva, I., Thor, M., Oates, E., Karnebeek, C., Hendson, G., Blom, E., Witting, N., Rasmussen, M., Gabbett, M., Ravenscroft, G., Sframeli, M., Sutterlin, K., Sarkozy, A., D’Argenzio, L., Hartley, L., Matthews, E., Pitt, M., Vissing, J., Bellegaard, M., Krarup, C., Slordhal, A., Halvorson, H., Ye, C., Zhang, L.H., Lokken, N., Werlauf, U., Abdelsayed, M., Davis, M.R., Feng, L., Phadke, R., Sewry, C.A., Morgan, J.E., Laing, N.G., Vallance, H., Ruben, P., Hanna, M.G., Lewis, S., Kamsteeg, E.J., Mannikko, R., Mutoni, F. (2016). Recessive loss-of-function SCN4A mutations associated with a novel phenotype of congenital myopathy, Brain, 139, 674-691.

68.Zapata-Wainberg, G., Gallego de la Sacristana, M., Vivancos, J. (2015). Canalopatías del músculo esquelético de base genética: parálisis periódicas y miotonías no distróficas, Medicine, 11(75), 4511-5. 
